\title{
Lectin binding sites in normal, scarred, and lattice dystrophy corneas ${ }^{\star}$
}

\author{
Department of \\ Ophthalmology, \\ University of \\ Manchester, Manchester \\ PN Bishop \\ A E A Ridgway \\ Department of \\ Pathological Sciences \\ P N Bishop \\ R E Bonshek \\ C J P Jones \\ R W Stoddart \\ Correspondence to: \\ Mr P N Bishop, Department \\ of Pathological Sciences, \\ Stopford Building, University \\ of Manchester, Manchester \\ M13 9PT. \\ Accepted for publication \\ 2 August 1990
}

\author{
P N Bishop, R E Bonshek, C J P Jones, A E A Ridgway, R W Stoddart
}

\begin{abstract}
Normal, scarred, and dystrophic corneas were histochemically probed with a panel of 16 lectins by means of an avidin-biotin revealing system. Normal corneal epithelial cells, keratocytes, and endothelial cells expressed at least two distinct $\mathbf{N}$-linked oligosaccharide subsets, of the non-bisected, biantennary and bisected, bi-/triantennary types. Corneal scars stained variably with the lectin subsets described above, and with Maclura pomifera agglutinin. Lattice dystrophy corneas showed a loss of the oligosaccharide expression observed on the plasma membranes of normal epithelial cells, and there was concurrent deposition of extracellular glycoprotein within the corneal stroma, which was of the same oligosaccharide subsets as were lost from the epithelial cell plasma membranes. This extracellular stromal glycoprotein was far more widely deposited than the amyloid and extended well beyond the stromal scarring. We propose that these observations are related and that in lattice corneal dystrophy a glycoprotein(s) is shed from the plasma membranes of epithelial cells and sequestrated within the corneal stroma, where it subsequently stimulates amyloid deposition.
\end{abstract}

Lattice corneal dystrophy type 1 is an autosomal dominant condition ${ }^{1}$ causing progressive bilateral corneal opacities typified by lattice lines. Histologically the lattice lines have the staining characteristics of amlyoid. ${ }^{2}$ Previous studies, including studies in lectin histochemistry, have demonstrated 'abnormal' extracellular material within the corneal stroma apart from the amyloid, ${ }^{34}$ while electron microscopic techniques have identified a similar 'abnormal' material between and beneath the basal epithelial cells. ${ }^{35}$ However, the relationship between this material, the amyloid deposits, and underlying cellular mechanisms has not been clearly defined.

As lectins are known to bind to the extracellular deposits of lattice corneal dystrophy, including the amyloid, ${ }^{4}$ and they have an affinity for cellular oligosaccharides, a panel of lectins was used histochemically to probe corneas with this condition. Normal corneas were examined, as their binding to the lectins used in this study was previously only partially defined. ${ }^{67} \mathrm{~A}$ pathological sequel of lattice corneal dystrophy is scarring. We therefore examined corneas with scarring arising from a variety of other causes, in

^ Previously presented as an abstract to the Pathological Society of Great Britain (see Bishop $e t a^{3}$ ), and some of the results were given in a lecture by A E A Ridgway entitled 'Corneal amyloid and Congress of Ophthalmology, Singapore, March 1990. order to establish the contribution of scarring to the observed lectin binding pattern in lattice corneal dystrophy.

\section{Materials and methods}

Four normal corneas were obtained from eyes enucleated for malignant melanoma of the choroid (patients' ages 38-73 years). Six scarred corneal buttons were obtained at penetrating keratoplasty following trauma, trachoma, interstitial keratitis, herpes simplex keratitis, and one with longstanding scarring of uncertain cause. Twelve corneal buttons were obtained from patients (aged 25-90 years), these were histologically diagnosed as lattice dystrophy corneas. Seven of these corneas were from four distinct pedigrees with the classical clinical signs and an autosomal dominant family history characteristic of type 1 lattice dystrophy. The other five histologically diagnosed buttons came from patients in whose case notes a family history was not recorded, so that it was not possible to give an unequivocal diagnosis of the type 1 variety.

All specimens were immediately fixed in $10 \%$ (v/v) neutral formalin buffered with $0.1 \mathrm{M}$ phosphate buffer, and cetylpyridinium chloride (CPC) $0 \cdot 1 \%(\mathrm{w} / \mathrm{v}), \mathrm{pH} 7 \cdot 4$, for 16 to 24 hours, prior to dehydration and embedding in paraffin wax. Each cornea underwent conventional histological examination, including staining with haematoxylin and eosin, Congo red, and the periodic acid-schiff (PAS) reaction.

For lectin histochemistry, sections were cut at $4 \mu \mathrm{m}$, mounted on poly-1-lysine coated slides, and treated with biotinylated lectin followed by avidin peroxidase conjugate. A biotinylated lectin is a lectin to which biotin has been synthetically coupled. The biotin can then act as a reporter molecule which is specifically detected by interaction with avidin or streptavidin. The avidin used here is chemically coupled to the enzyme horseradish peroxidase, which in turn is revealed by interaction with DAB (diaminobenzidine tetrahydrochloride dihydrate). This revealing system is extremely sensitive and specific, ultimately resulting in the dense brown stain of DAB corresponding to areas of lectin binding.

Biotinylated lectins, avidin peroxidase conjugate, and crude trypsin (type II from porcine pancreas), were obtained from Sigma, Poole, Dorset, UK. The lectins used in this study were those of Jack bean (Canavalia ensiformis, con A), garden pea (Pisum sativum, PSA), common lentil (Lens culinaris, LCA), kidney bean (Phaseolus vulgaris, type III, erythroagglutinin, e-PHA, and type IV leucoagglutinin, 1-PHA), wheat germ (Triticum vulgaris, WGA), pokeweed (Phytolacca 
Table 1 Lectin staining in normal and lattice dystrophy corneas: comparison of basal epithelial cells and stroma (averaged results for all normal and lattice dystrophy corneas)

\begin{tabular}{|c|c|c|c|c|c|c|}
\hline & \multicolumn{6}{|l|}{ Lectins } \\
\hline & $\operatorname{Con} A$ & $P S A$ & $L C A$ & $e P H A$ & $W G A$ & $M P A$ \\
\hline \multicolumn{7}{|l|}{$\begin{array}{l}\text { Normal Comea } \\
\text { Basal epithelial cells }\end{array}$} \\
\hline Plasma membrane & ++ & +++ & +++ & +++ & +++ & - \\
\hline Cytoplasmic granules & +++ & ++ & ++ & ++ & ++ & - \\
\hline Nuclear envelope & ++ & - & - & - & - & - \\
\hline Cytoplasm background & + & $+1-$ & $+1-$ & - & - & - \\
\hline Stroma & & & & & & \\
\hline \multirow{2}{*}{\multicolumn{7}{|c|}{$\begin{array}{l}\text { Lattice dystrophy cornea } \\
\text { Basal epithelial cells`}\end{array}$}} \\
\hline & & & & & & \\
\hline Plasma membrane & $+1-$ & $+1-$ & $+1-$ & $+1-$ & $+1-$ & - \\
\hline Cytoplasmic granules & ++ & + & + & + & + & - \\
\hline Nuclear envelope & ++ & - & - & - & - & - \\
\hline Cytoplasm background & + & $+1-$ & $+1-$ & - & - & - \\
\hline \multicolumn{7}{|l|}{ Stroma } \\
\hline Background & + & + & + & - & - & - \\
\hline Extracellular deposits & +++ & +++ & +++ & +++ & ++ & - \\
\hline Amyloid (lattice lines) & + & + & + & + & + & - \\
\hline Scarring (where stained) & +++ & +++ & +++ & +++ & $++t$ & + \\
\hline
\end{tabular}

Scoring: absent $=-$, faint or equivocal $=+1-$, up to heavy staining $=+++$ * Excluding areas with disrupted epithelial architecture, see text. + Extracellular deposits excluding amyloid and scarring.

americana, PAA), soybean (Glycine max, SBA), peanut (Arachis hypogaea, AHA), hairy vetch (Vicia villosa, VVA), Bandeiraea simplicifolia isolectin 1-B4 (BSA), asparagus or winged pea (Tetragonolobus purpureus, LTA), gorse (Ulex europaeus isolectin 1, UEA), osage orange (Maclura pomifera, MPA), horse gram (Dolichos biflorus, DBA) and edible snail (Helix pomatia, HPA). All were used as biotinylated derivatives, and showed competitive inhibition with appropriate monosaccharides, apart from e-PHA and 1-pHA, which do not show monosaccharide inhibition.

A standard lectin staining procedure was used. The sections were dewaxed in xylene and then transferred to absolute alcohol. Endogenous peroxidase activity was blocked with $0 \cdot 5 \%(\mathrm{v} / \mathrm{v})$ hydrogen peroxide in absolute methanol containing $0.4 \%(\mathrm{v} / \mathrm{v}) 1 \mathrm{~N}$ hydrochloric acid for 30 minutes at $18^{\circ} \mathrm{C}$, and then the sections were washed in $0.05 \mathrm{M}$ Tris buffered saline (TBS), $\mathrm{pH}$ 7.6. Trypsinisation was carried out at $37^{\circ} \mathrm{C}$ for 10 minutes with crude trypsin (Sigma type II), to expose sugar residues occluded by fixation. ${ }^{10}$ After being washed in TBS the sections were incubated at $18^{\circ} \mathrm{C}$ with $10 \mathrm{mg} / \mathrm{l}$ biotin labelled lectin in $0.05 \mathrm{M} \mathrm{TBS}, \mathrm{pH} 7.6$, with $1 \mathrm{mM} \mathrm{CaCl}_{2}$ for 30 minutes. Control sections were included in each run, which were treated identically apart from being incubated in buffer alone at this stage. All sections were washed in TBS containing $1 \mathrm{mM} \mathrm{CaCl} 2$ and placed in $5 \mathrm{mg} / \mathrm{l}$ avidinperoxidase in $0.125 \mathrm{M}$ TBS (containing $0.347 \mathrm{M}$ $\mathrm{NaCl}$ ) for 1 hour. The sections were jet washed in TBS $(0.05 \mathrm{M})$ and incubated in a solution of 0.05\% (v/w) DAB (Aldrich Chemical Co., Gillingham, UK), 0.05 M TBS and 0.015\% (v/v) hydrogen peroxide for 5 minutes at room temperature. They were counterstained with $0.25 \%(\mathrm{w} / \mathrm{v})$ methyl green in $0.1 \mathrm{M}$ sodium acetate buffer ( $\mathrm{pH} 4 \cdot 8$ ), before being dehydrated and mounted in DePeX. Photography was performed on an Olympus BH-2 photographic microscope, with an Olympus OM PM-10AD photomicrographic system. The film used was Kodak Panatomic-X.

\section{Results}

Each group of corneas (normal, scarred, and lattice dystrophy) produced consistent lectin binding patterns, except where stated. The four

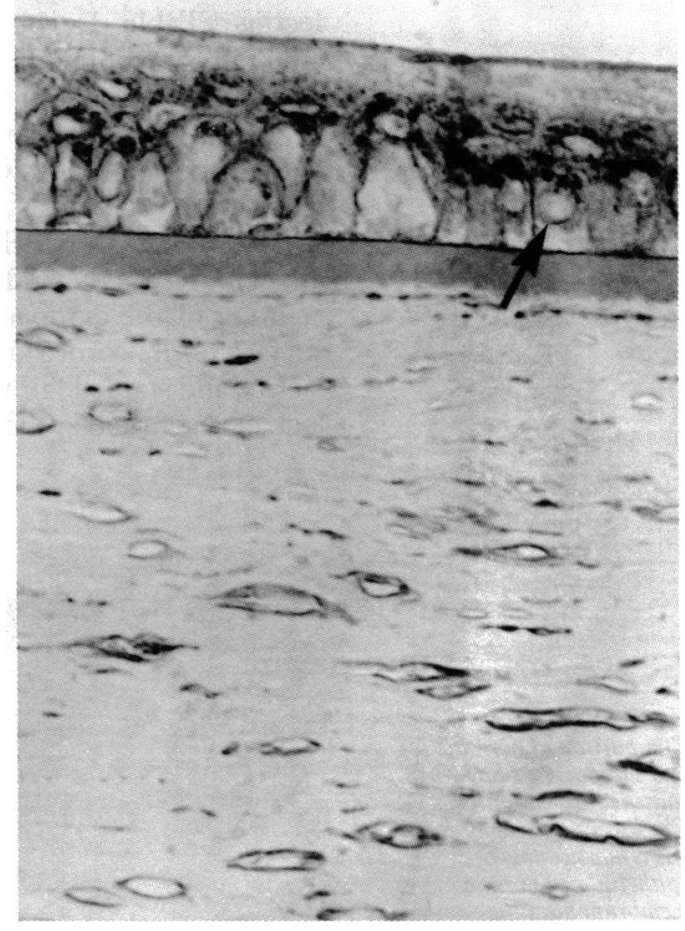

Figure 2 Normal cornea stained with con A. Same section as Fig 1 showing similar staining to $L C A$, but also staining of epithelial nuclear envelope (arrow) and diffuse cytoplasmic staining. $(\times 280$.

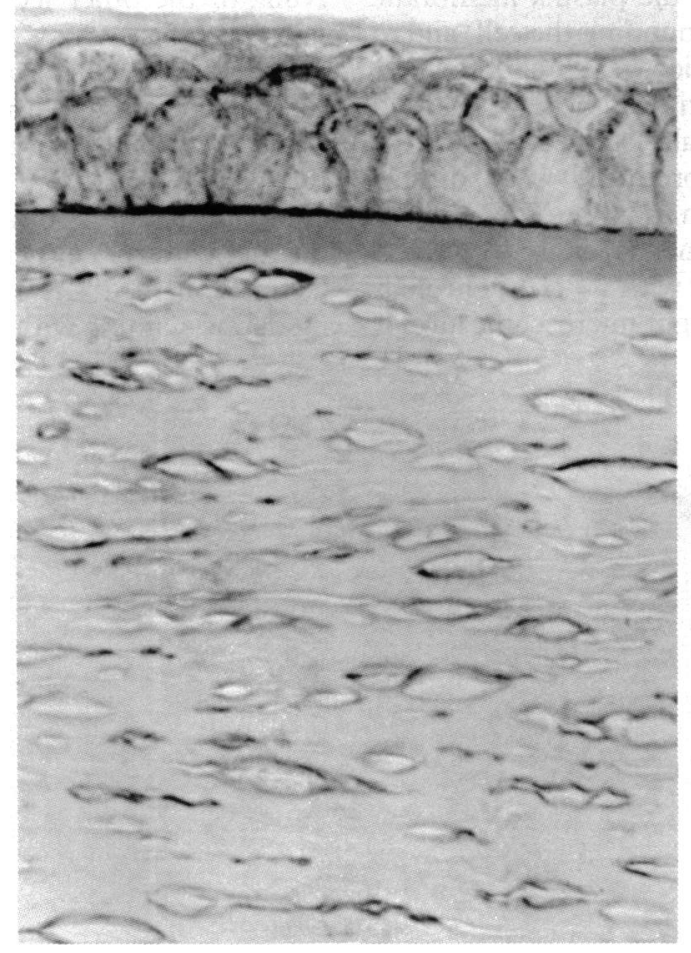




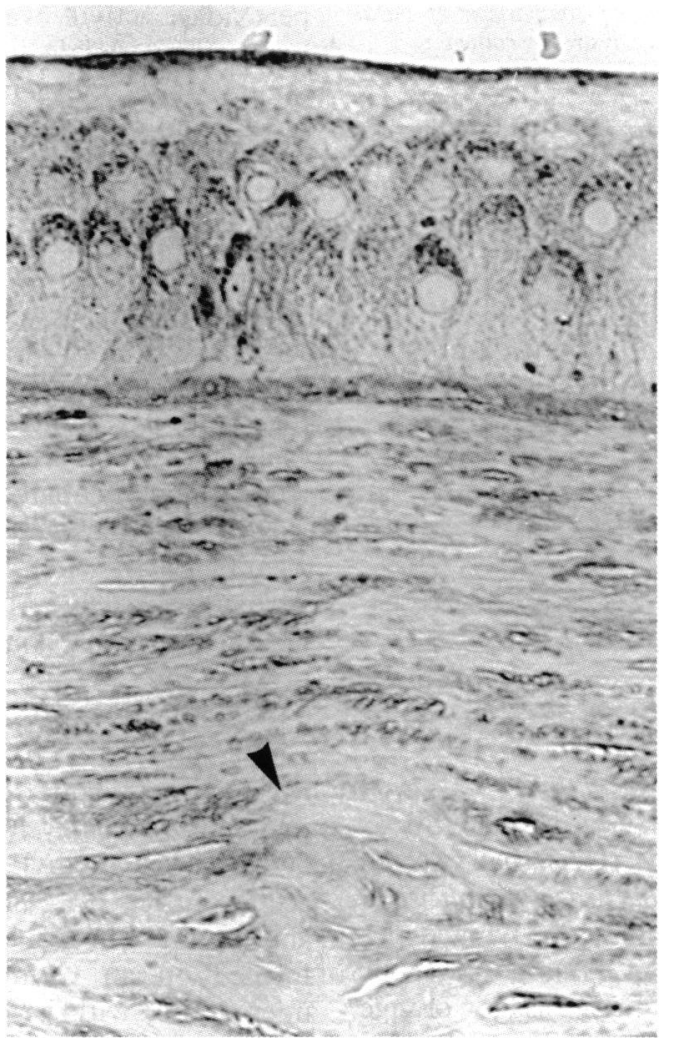

Figure 3 Lattice dystrophy cornea stained with con A. There is a loss of epithelial cell plasma membrane (surface

membrane) staining highlighted by preservation of cytoplasmic granular staining. Nuclear envelope staining is preserved. The anterior stroma has extensive densely staining extracellular stromal deposits and also a lightly staining lattice line which is difficult to distinguish above normal background staining (arrow). $(\times 560$. $)$

normal corneas all showed staining of their epithelial cells, keratocytes, and endothelial cells with the lectins con A, PSA, LCA, e-PHA, and WGA. The epithelium had strong staining of its basal and inner wing cell layers with all of these lectins (Table 1), but had progressively less staining of the more superficial layers. The staining was localised to the plasma membrane (the surface membrane bounding the cell) and to cytoplasmic granules, which were predominantly apical in the basal cells, with all these lectins (Fig 1), except con A, which also stained the nuclear envelope strongly and produced weak diffuse staining of the cytoplasm (Fig 2). The anterior surface of the cornea stained strongly with these lectins and stained faintly with 1-PHA. Through lack of resolution at the light microscopic level it

Figure $5 \quad A, B$ Comparison of normal $(A)$ and lattice dystrophy $(B)$ corneas stained with con $A$. The lattice dystrophy cornea has a marked loss of epithelial cell plasma membrane staining, with a concurrent deposition of stainable extracellular material within the anterior stroma. $(\times 32$.

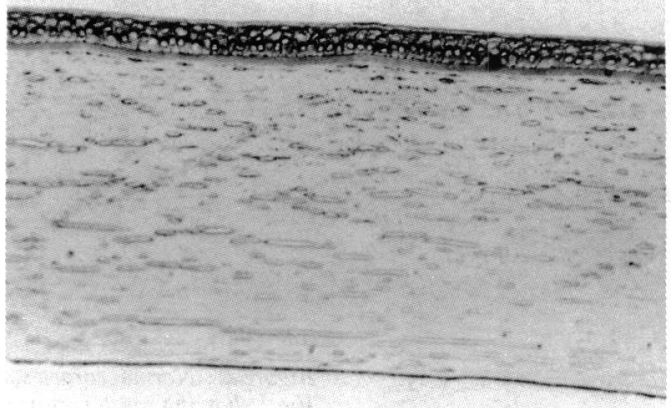

Figure $5 A$

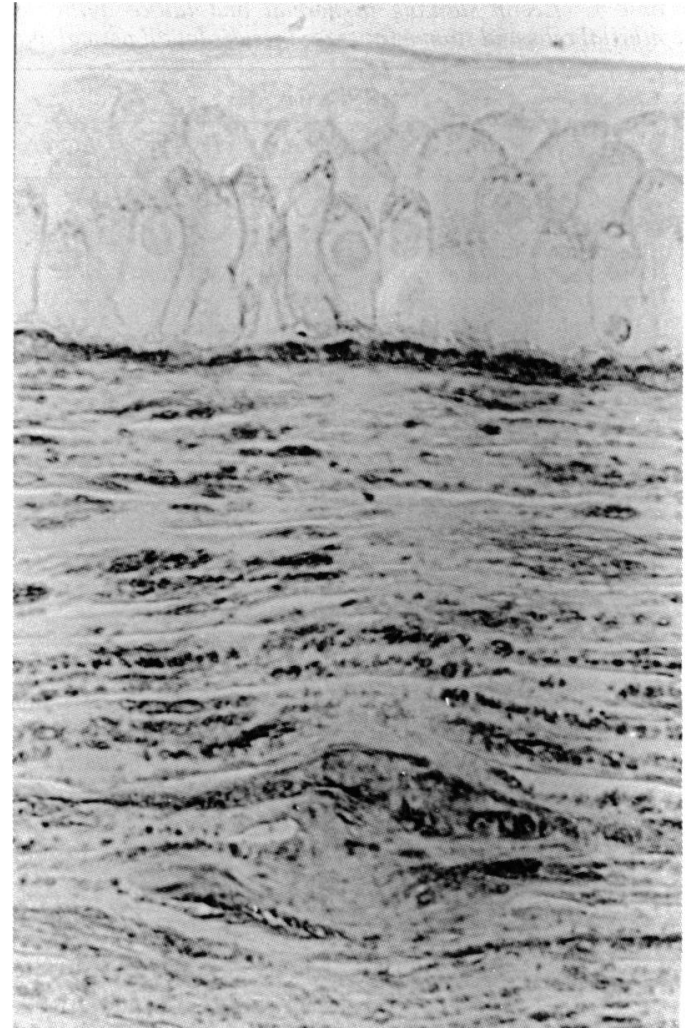

Figure 4 Lattice dystrophy cornea stained with PSA. Same specimen as Fig 3, with marked reduction in epithelial cell plasma membrane staining and reduced, but still visible cytoplasmic granular staining. $(\times 560$.)

was difficult to determine the distribution of staining within the keratocytes and endothelial cells. The normal corneal epithelial cell basement membrane and Bowman's membrane variably stained with con A, PSA, LCA, e-PHA, and WGA. This staining, when present, was pale and often inconstant along the length of the membranes. The corneal stroma demonstrated diffuse pale staining with con A, PSA, and LCA. No staining of Descemet's membrane was observed. None of the other lectins in the panel stained normal corneas.

All six scarred corneas had a similar lectin binding pattern to normal corneas, except in the zones of stromal scarring and, sometimes, in localised areas of overlying disorganised epithelium. Stromal scar tissue showed two distinct patterns, either heavy staining with the lectins con A, PSA, LCA, e-PHA, WGA and moderate staining with the lectin MPA, or zones

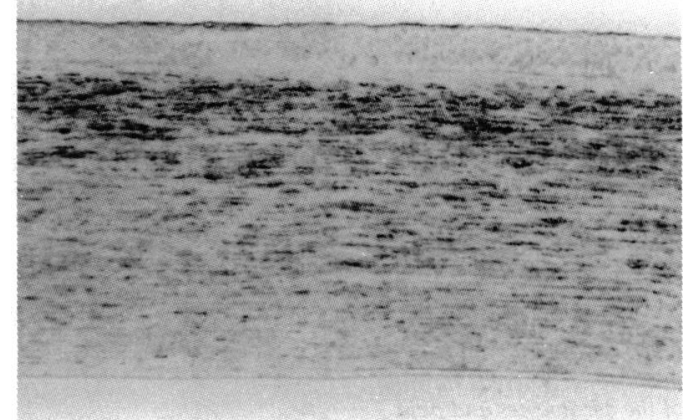

Figure 5B 


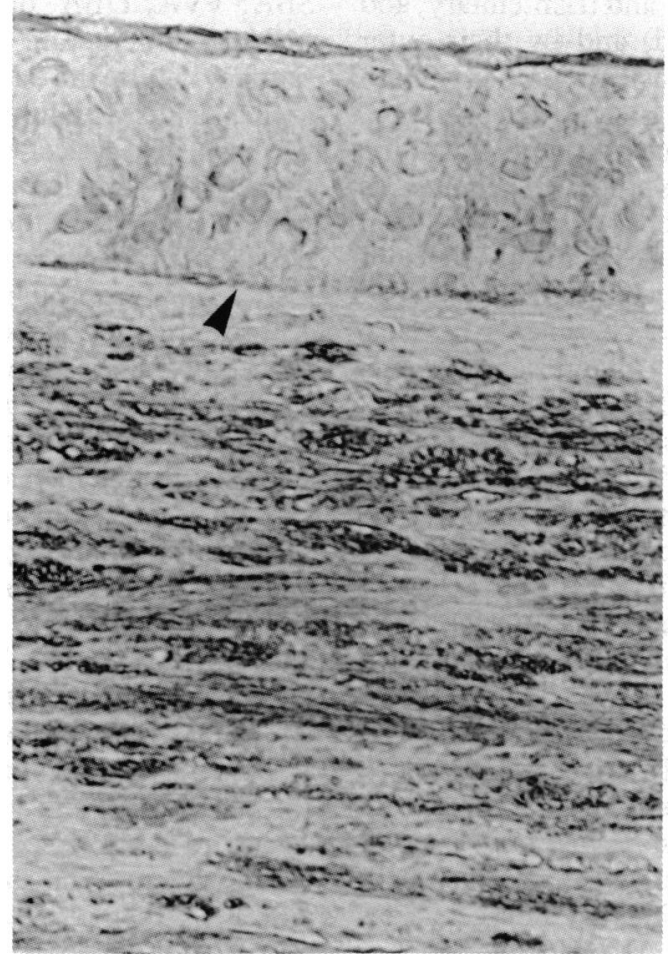

Figure 6 Lattice dystrophy cornea stained with con $A$. This shows similar features to Fig 4, with preservation of staining of the nuclear envelope but a more marked loss of cytoplasmic granular staining. $A$ band of staining is clearly visible superficial to the epithelial basement membrane (arrow). (×420.)

where the lectin staining was absent. These unstained zones corresponded to hyalinised patches seen on haematoxylin and eosin staining. The overlying epithelium showed inconstant lectin labelling, which was variably normal, increased, or absent. If staining was absent there was a total loss of lectin binding, including the binding of con $A$ to the nuclear envelope.

All twelve corneas with lattice corneal dystrophy showed a similar lectin binding pattern. They gave morphological evidence of superficial stromal scarring - that is, disruption of the collagenous architecture - which produced the same staining patterns as observed in the group of scarred corneas, including focal MPA staining when scarring was prominent. The amyloid of the lattice lines stained with the lectins con A, PSA, LCA, e-PHA, and WGA (Table 1). This staining was generally weak and diffuse (Figs 3 and 4). All the dystrophic samples had extensive extracellular staining of the corneal stroma, which was most dense anteriorly and centrally, including and extending beyond the areas of scarring and amyloid deposition (Figs 3, 4, and 5A, B). Similar extracellular staining was variably seen between the basal epithelial cells and their basement membrane and between the basement membrane and Bowman's membrane (where the latter was preserved). In some instances this staining was of thickly deposited masses of extracellular material (Figs 3, 4, 6); such a pattern was never seen in corneal buttons with scarring alone.

The epithelial plasma membranes of lattice dystrophy corneas showed a generalised loss of staining as compared with normal corneas (which stained with con A, PSA, LCA, e-PHA, and WGA). There was, however, preservation of staining of cytoplasmic granules (albeit reduced) (Figs 3 and 4) and the nuclear envelope staining with con A (Figs 3 and 6) (Table 1). This contrasted with the complete loss of staining of all cellular components seen in the disorganised epithelium of some corneas with scarring alone. The anterior corneal surface, Descemet's membrane, and the endothelium all had the same staining characteristics as normal cornea. Keratocytes were difficult to identify because of the dense stromal staining.

It was notable that, in lattice dystrophy corneas, the loss of staining of architecturally normal epithelium was accompanied by an accumulation of stainable material within the subjacent regions of corneal stroma and immediately above and below the epithelial basement membrane. This stainable material extended well beyond any anterior stromal scarring and associated overlying epithelial disorganisation, which was variably seen in these specimens.

None of the corneas studied (normal, scarred, or lattice dystrophy) showed staining with the lectins PAA, SBA, AHA, VVA, BSA, LTA, UEA, DBA, and HPA.

\section{Discussion}

Corneas with lattice corneal dystrophy frequently show at least incipient scarring, hence the necessity to make comparisons with corneas scarred from other causes as well as normal corneas. The features which were peculiar to dystrophic corneas and which could not be attributed to scarring were: (1) extensive reduction, or total loss, or staining of epithelial cell plasma membranes, without marked loss of staining of their contents; (2) coextensive increase in staining of the superficial corneal stroma with, in some cases, the appearance of amorphous, stained deposits above and beneath the epithelial basement membrane; and (3) deposition of focal amyloid deposits within the corneal stroma. Stromal scarring, however, though associated with similar lectin staining (apart from also staining with MPA), had a different distribution and no clear association with epithelial change. The corneal epithelium superficial to scarring sometimes showed a highly focal loss of staining associated with architectural disruption. This involved all intracellular structures and may represent a focal loss of permeability to macromolecular reagents.

Lectins are proteins or glycoproteins, usually of plant origin, which have, the property of binding to specific subsets of oligosaccharides (short sugar chains, which if $\mathrm{N}$-linked contain about 12 to 24 monosaccharide units). Oligosaccharides are the saccharide component of 'classical' glycoproteins, glycolipids, and are present on core proteins of proteoglycans. Oligosaccharides destined for the cell plasma membrane and extracellular matrix components are variously synthesised within the endoplasmic reticulum and then Golgi apparatus of the cell." They are broadly divided into two groups based on the chemical structure of the protein/sugar linkage region. These are the $\mathrm{N}$-linked and $\mathrm{O}-$ linked varieties. The $\mathrm{N}$-linked oligosaccharides can then be further defined by their typical types 
of branching structure (bi- and triantennary, and bisected and non-bisected) and by their outer monosaccharide sequences (the inner monosaccharide sequences being relatively constant).

From the known binding characteristics of the panel of lectins used in this study, some tentative conclusions as to oligosaccharides expressed can be derived, as follows. PSA and LCA bind strongly to non-bisected, bi- or triantennary, complex, $\mathrm{N}$-linked oligosaccharides. They also bind more weakly to high mannose and intermediate, non bisected, $\mathrm{N}$-linked oligosaccharides. ${ }^{12-15}$ Con A binds to high mannose, intermediate, and small biantennary, complex $\mathrm{N}$-linked oligosaccharides whether bisected or not. ${ }^{1316-19}$ WGA has more varied binding capabilities, since it can interact strongly with di$\mathrm{N}$-acetyl chitobiosyl sequences, some $\mathrm{N}$-acetyl lactosamine sequences and, more weakly, with some sialyl residues. ${ }^{20-23}$

1-PHA binds selectively to tri- and tetraantennary, non-bisected oligosaccharides. ${ }^{134}$ We failed to demonstrate any binding with 1-PHA (apart from faint staining of the anterior corneal surface), but binding was observed with PSA and LCA, thus implying that the saccharides that bind PSA and LCA are probably biantennary and not triantennary. A similar phenomenon has been found in mast cell granules. ${ }^{2526} \mathrm{e}-\mathrm{PHA}$ has an obligate requirement for bisected bi- or triantennary oligosaccharides ${ }^{2427}$ and therefore binds to different structural subsets from PSA, LCA, and 1-PHA. e-PHA has some overlap with con A. None of the corneas examined had evidence of highly branched $\mathrm{N}$-linked saccharides, except at the anterior corneal surface.

The lack of staining with the other lectins in the panel implies that L-fucose, $\alpha-\mathrm{D}-\mathrm{N}$ acetylgalactosamine and the $\alpha$ and $\beta$ anomers of $D$-galactose were generally absent as nonreducing terminal sugars, either in N-linked or $\mathrm{O}$-linked saccharides. Because $\mathrm{N}$-linked saccharides were demonstrably present, the implication is that they were probably terminally sialylated. While there was no direct evidence for the presence of $\mathrm{O}$-linked saccharides, their occurrence could not be excluded.

The normal corneal epithelial plasma membrane and cytoplasmic granules expressed at least two subsets of $\mathrm{N}$-linked oligosaccharides. These were the non-bisected bi-antennary and the bisected bi-/triantennary types. The predominance of oligosaccharide expression on the basal epithelial cells has previously been demonstrated in normal rat corneas. ${ }^{28}$ The keratocytes and endothelial cells carried similar oligosaccharide subsets. The nuclear envelope and cytoplasm of the epithelial cells specifically expressed putative 'high mannose' saccharides. The extracellular matrix of the normal corneal stroma also expressed $\alpha$-D-mannose particularly in the form of bi-/triantennate $\mathrm{N}$-linked complexes. This may represent binding to proteoglycan core, collagen, or collagen-related oligosaccharides.

Corneal stromal scars expressed non-bisected biantennary and bisected bi-/triantennary saccharides. They also stained with MPA which has an affinity for $\alpha$-D-galactose, and $\mathrm{N}$-acetyl $\alpha$ D-galactosamine. ${ }^{29}$ Since there was no binding of
SBA, VVA, DBA, or HPA, it is unlikely that MPA was interacting with $\alpha$ - $\mathrm{N}$-acetyl-D-galactosamine, while the lack of staining with BSA argues against a binding via $\alpha$-D-galactose (though we have observed a similar disparity in other tissues). The precise mechanism of the staining with MPA is therefore uncertain, though it is possibly binding to a saccharide on collagen or an associated glycoprotein of corneal scar tissue.

In the dystrophic corneas the lectins showed reduced or absent binding to the epithelial cell surfaces, thus implying defective synthesis or loss of plasma membrane oligosaccharide. The same lectins, and hence the same oligosaccharide subsets, were expressed in deposits within the subjacent stroma, suggesting, but not proving, that the two phenomena might be related. Panjwani $\mathrm{et} \mathrm{al}^{4}$ found a similar stromal binding pattern with con A, WGA, and RCA-1 using paraffin section, but did not comment on epithelial lectin binding.

We suggest that glycoprotein is shed from the epithelial cells and then passes through the cornea to become sequestrated, predominantly within the anterior corneal stroma. The glycoprotein is retarded by the epithelial basement membrane, which is known to act as a semipermeable filter ${ }^{30}$ and Bowman's layer, resulting in the accumulation of stainable material at these anatomical interfaces. We further hypothesise that the glycoprotein, or some fragment of it, subsequently stimulates focal amyloidogenesis (amyloid production essentially involves focal nidation which once started is self perpetuating). ${ }^{31}$ It is likely that this step involves the handling of the glycoprotein by keratocytes with subsequent injury to them, as intracytoplasmic material has been demonstrated within keratocytes adjacent to the amyloid deposits, ${ }^{25}$ and degenerate keratocytes are reported in these areas. $^{2}$ Some part of the amyloid deposit could derive from the keratocytes rather than the epithelial glycoconjugate, since corneal scars of various origins can occasionally contain amyloid. ${ }^{32}$

This hypothesis accords with the clinical characteristics of type 1 lattice corneal dystrophy. It begins in childhood, the first clinical sign being subepithelial corneal opacities. ${ }^{33}$ There is usually a history of recurrent corneal erosions at this stage, suggesting an early defect at the level of the corneal epithelial plasma membrane or basement membrane. Later a diffuse anterior stromal haze develops ${ }^{33}$ which may represent the diffusion of glycoprotein into the corneal stroma and the extensive extracellular lectin staining seen in lattice dystrophy corneas. All these changes occur well before the classical amyloid-containing stromal lattice lines appear, suggesting that the deposition of amyloid is not a primary phenomenon. Amyloidogenesis within the corneal stroma is an occasional response to a variety of insults, including trichiasis, previous trauma, keratoconus, and granular corneal dystrophy. ${ }^{32+36}$ In lattice corneal dystrophy the abnormal presence of a diffusible glycoprotein, derived from the corneal epithelial plasma membranes, may be another such insult, but one which results in a particularly marked degree of amyloid deposition. 
This research was supported by The North West Regional Health Authority, of Great Britain.

1 Kivlin J, Lovrien EW, Maumenee IH, Bishop DT, Bias W Linkage analysis in lattice corneal dystrophy. Am $\mathcal{J} M e d$ Genet 1984; 19: 387-90.

2 Klintworth GK. Lattice corneal dystrophy. An inherited variety of amyloidosis restricted to the cornea. $A m \mathcal{F}$ Pathol 1967; 50: 371-99.

3 de Felice GP, Carta S. Lattice dystrophy of the cornea. A clinical and histopathological study. Ophthalmologica 1986; 192; 135-42.

4 Panjwani N, Rodrigues M, Free K, Krachmer JH, Albert D, Baum J. Lectin receptors of amyloid in corneas with lattice Baum J. Lectin receptors of amyloid in corneas
dystrophy. Arch Ophthalmol 1987; 105: 688-91.

5 Wittebol-Post D. Corneadystrofieën Op 'Nieuw' Belicht. Dordrecht: ICG Printing, 1987: 135-42.

6 Pajwani N, Moulton P, Alroy J, Baum J. Localisation of lectin binding sites in human, cat, and rabbit corneas. Invest Ophthalmol Vis Sci 1986; 27: 1280-4.

7 Brandon DM. Nayak SK, Binder PS. Lectin binding patterns of the human cornea. Comparison of frozen and paraffin sections. Comea 1988; 7: 257-66.

8 McMahon RFT, Benbow EW, Lofthouse AK, Stoddart RW. Human salivary gland glycoconjugates: a lectin histochemical study. Histochem $\mathcal{F} 1989 ; 21: 663-74$.

9 Jones CJP, Mosley SM, Jeffrey IJM, Stoddart RW. Elimination of non-specific binding of avidin to tissue sections. Histoof non-specific binding

10 Jeffrey IJM, Mosley SM, Jones CJP, Stoddart RW. Proteolysis and lectin histochemistry. Histochem $\mathcal{F} 1987 ; 19: 269-75$.

11 Darnell J, Lodish H, Baltimore D. Molecular cell biology. New York: Scientific American Books, 1986: 957-64.

12 Trowbridge IS. Isolation and chemical characterisation of a mitogenic lectin from Pisum sativum. I Biol Chem 1984; 249: 6004-12.

13 Debray H, Decont D, Strecker G, Spik G, Montreuil J. Specificity of twelve lectins towards oligosaccharides and glycopeptides related to N-glycosylproteins. Eur $\mathcal{f}$ Biochem 1981; 117: 41-55.

14 Kornfeld K, Reitman ML, Kornfeld R. The carbohydrate binding specificity of pea and lentil lectins. Fucose is an important determinant. $₹$ Biol Chem 1981; 256: 6633-40.

15 Debray $\mathrm{H}$, Montreuil J. Structural basis for the affinity of four insolubilised lectins, with a specificity for $\alpha$-D-mannose, towards various glycopeptides with the $\mathrm{N}$-glycosylamine towards various glycopeptides with the $\mathrm{N}$-glycosylamine linkage

16 Goldstein IJ, Hayes CE. The lectins: carbohydrate binding proteins of plants and animals. Adv Carbohydr Chem Biochem 1978; 35: 128-340.

17 Goldstein IJ, Iyer RN, Smith EE, So LL. Protein carbohydrate interaction XX. The interaction of concanavalin A with sophorose and some of its derivatives. Biochemistry 1967 6: 2373-7.

18 Goldstein IJ, Cifonelli JA, Duke J. Interaction of concanavalin
A with capsular polysaccharide of the pneumococcus type XII and isolation of kojobiose from the polysaccharide. Biochemistry 1974; 13: 867-70.

19 Kornfeld R, Ferris C. Interaction of immunoglobulin glycopeptides with concanavalin A. F Biol Chem 1975; 250: peptides

20 Allen AK, Neuberger A, Sharon N. The purification, composition, and specificity of wheat germ agglutinin. Biochem $\mathcal{F}$ 1973; 131: 155-62.

21 Bhavanandan VP, Katlic AW. The interaction of wheat germ agglutinin with sialoglycoprotein. F Biol Chem 1979; 254 agglutinin

22 Gallagher JT, Harding M, Dale RE. Sialic acid as a cell surface binding site for wheat germ agglutinin. In: Bog-Hansen TC. Spengler GA, eds. The lectins: biology, biochemistry, clinical biochemistry. Berlin and New York: Walter de Gruyter, 1983; 3: 311-8.

23 Gallagher JT, Morris A, Dexter TM. Identification of two binding site for wheat germ agglutinin on polylactosamine type oligosaccharides. Biochem $f$ 1985; 231: 115-22.

24 Cummings RD, Kornfeld S. Characterisation of the structura determinants required for the high affinity interactions of asparagine-linked oligosaccharides with immobilised Phaseolus oulgaris leucoagglutinating and erythro agglutinating lectins. $f$ Biol Chem 1982; $257: 11230-4$.

25 Stoddart RW, Roberts ISD. Interaction between mast cells and fibroblasts in neurofibroma: a commentary. Pathol Res and fibroblasts in neurofibn

26 Roberts ISD, Jones CJP, Stoddart RW. Lectin histochemistry of the mast cell: heterogeneity of rodent and human mast cell of the mast cell: heterogeneity of rodent and

27 Yamashita AK, Nitoi A, Kobata A. Structural determinants of Phaseolus vulgaris erythroagglutinating lectin for oligosaccharides. F Biol Chem 1983; 258: 14753-5.

28 Gipson IK, Riddle C, Kiorpes T, Spurr S. Lectin binding to cell surfaces: comparisons between normal and migrating corneal epithelium. Dev Biol 1983; 96: 337-45.

29 Bausch JN, Poretz RD. Purification and properties of the haemagglutinin from Maclura pomifera seeds. Biochemistry 1977; 16: 5790-4.

30 Kefalides NA, Alper R, Clark CC. Biochemistry of basement membranes. Int Rev Cytol 1979; 61 : 167.

31 Bishop PN, Jones CPJ, Stoddart RW. An abnormal saccharide distribution in lattice corneal dystrophy. F Pathol 1989; 157 : distribution in lattic

32 McPherson S, Kiffney GT, Freed CC. Corneal amyloidosis. Am f Ophthalmol 1966; 62: 1025-33.

33 Dubord P, Krachmer J. Diagnosis in early lattice corneal dystrophy. Arch Ophthalmol 1982; 100: 788

34 Garner A. Amyloidosis of the cornea. Br $\mathcal{F}$ Ophthalmol 1969 53: 73-81.

35 Stern GA. Knapp A, Hood CI. Corneal amyloidosis associated with keratoconus. Ophthalmology 1988; 95: 52-5.

36 Folberg R, Alfonso E, Croxatto JO et al. Clinically atypical granular corneal dystrophy with pathologic features of lattice-like amyloid deposits. Ophthalmology 1988; 95: 46-51. 